\title{
Recent Advances in Birefringence Studies at THz Frequencies
}

\author{
Karin Wiesauer • Christian Jördens
}

Received: 4 February 2013 / Accepted: 4 April 2013 /

Published online: 9 May 2013

(C) The Author(s) 2013. This article is published with open access at Springerlink.com

\begin{abstract}
In the last few years, various studies on terahertz $(\mathrm{THz})$ birefringence have been presented, based on polarization-sensitive $\mathrm{THz}$ time-domain spectroscopy. The field of $\mathrm{THz}$ birefringence is a wide one covering several aspects, such as different materials exhibiting $\mathrm{THz}$ birefringence, polarization-sensitive $\mathrm{THz}$ technology, as well as numerous methods for extracting the birefringence properties of a sample from the $\mathrm{THz}$ data. Therefore, this paper aims at reviewing recent results on $\mathrm{THz}$ birefringence measurements presented in literature, addressing the above aspects and giving an overview of the topic. Moreover, it will focus on the investigation of birefringent fibrous samples, where specific results will be discussed in detail. Altogether, this paper should give a comprehensive view on the recent achievements in the measurements of $\mathrm{THz}$ birefringence.
\end{abstract}

Keywords Terahertz $\cdot$ Birefringence $\cdot$ Anisotropy $\cdot$ Polarization-sensitive

\section{Introduction}

Birefringence is a well-known phenomenon at optical frequencies, but is also observed for various materials in the $\mathrm{THz}$ spectral range. Numerous studies have been performed where $\mathrm{THz}$ birefringence measurements were conducted for the characterization of a sample, giving insight into its anisotropic material properties. In this paper, advances in birefringence studies at $\mathrm{THz}$ frequencies will be reviewed, based on polarization-sensitive (PS) THz timedomain spectroscopy (TDS). The reader is assumed to be familiar with the principles of THz-TDS or can refer to, e.g., [1]. Starting with a short general introduction to birefringence and its measurement in Chap. 2, an arc will be spanned from materials that were found to be birefringent for THz waves (Chap. 3), over the techniques used in PS-THz-TDS (Chap. 4),

K. Wiesauer $(\square)$

RECENDT Research Center for Non-destructive Testing GmbH, 4040 Linz, Austria

e-mail: karin.wiesauer@recendt.at

C. Jördens

Institut für Hochfrequenztechnik, Technische Universität Braunschweig, 38106 Braunschweig, Germany 
to several aspects in recent advances in THz birefringence studies (Chap. 5). Chapter 5.4 will especially focus on the investigation of birefringent fibrous materials by PS-THz-TDS, where in particular the three works given in [2-4] will be reviewed in detail. With no claim to completeness, this paper should give a comprehensive view of the field of THz birefringence measurements, also providing insight into its potential for material characterization. Another wide and prosperous field is PS-THz imaging, where numerous advances have been made in the last few years (see, e.g., [4-10]). Although the one or other aspect will be addressed marginally in the course of this paper, PS-THz imaging is not in its main focus and is worth being reviewed elsewhere. Another topic of its own relying on PS-THz techniques is THz ellipsometry (e.g., [11-14]), which will also not be addressed in this paper, however, clearly deserves close attention for material characterization.

\section{Birefringence}

Birefringence arises from electrically anisotropic properties of a material. The probably best known example for birefringence at optical frequencies is the double images obtained by a calcite crystal, first described by the Danish scientist Bartholin in 1669 [15]. Also intrinsically isotropic materials can show birefringent properties when anisotropy is induced, such as by mechanical deformation, also referred to as stress birefringence (e.g., [16]), by an electrical field due to the Pockels [17] or the electro-optic Kerr effect [18], or by the alignment of polar molecules. The latter is exploited in liquid crystals (e.g. [19]), which are widely used, such as for optical displays. In addition, form birefringence can arise from anisotropies due to aligned sub-wavelength structures of isotropic material (e.g., [20]).

Birefringent materials exhibit a refractive index that depends on the polarization and propagation direction of the probing light. Considering a uniaxially birefringent material, a ray incident at an arbitrary angle with regard to the optical axis is split into two linearly polarized rays: the ordinary ray, which obeys Snellius' law and which travels with the same velocity in every direction through the crystal according to a refractive index $n_{o}$, and the extraordinary ray, travelling with a velocity dependent on the propagation direction, according to a refractive index that lies in between $n_{e}$ and $n_{o}$. Here, $n_{e}$ and $n_{o}$ denote the refractive indices along the extraordinary and ordinary axis of the material, respectively, and determine its birefringence $\Delta n$ according to

$$
\Delta n=n_{e}-n_{o} .
$$

For $n_{e}>n_{o}$ one talks of positively, in the other case of negatively birefringent materials. Figure 1 shows the refractive index ellipsoid of a positively birefringent material, where the major and the minor axis are given by $n_{e}$ and $n_{o}$, respectively. $O A$ denotes the direction of the optical axis, oriented by an angle $\theta$ with respect to the horizontal.

Depending on the birefringence and thickness $d$ of the material and the wavelength $\lambda$ of the probing light, a phase shift $\Delta \varphi$ is introduced between the ordinary and the extraordinary ray according to

$$
\Delta \varphi=\frac{2 \pi}{\lambda} d \Delta n
$$

This effect is commonly exploited for the fabrication of half or quarter wave plates exhibiting phase shifts of $\pi$ or $\pi / 2$. As birefringence influences the polarization vector of an 


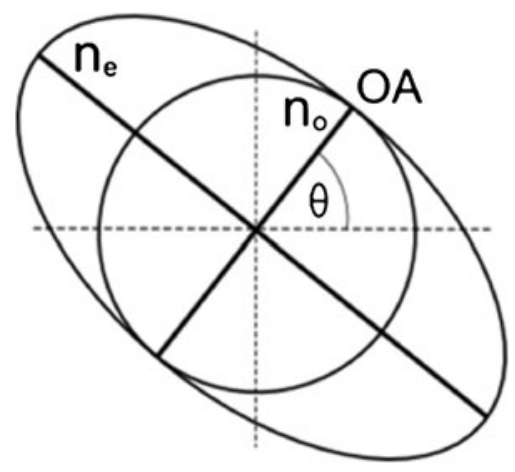

Fig. 1 Refractive index ellipsoid of a uniaxial, positively birefringent material

electromagnetic wave passing through the sample in a deterministic way, its measurement can be used to extract information about material properties. Besides birefringence in terms of linearly polarized radiation as considered in this paper, also optical activity is present in many media, which is described by different refractive indices for left and right circularly polarized light. The details are discussed elsewhere and can be found in common optics textbooks.

Classically, birefringence is probed by various polarization optical methods, where the effect of the sample on the polarization state of the probing light is investigated. One established technique is photoelasticity (e.g., [21]), where transparent models of mechanically stressed components are imaged by polarized light, and highly stressed regions become visible. A more specialized method is ellipsometry (e.g., [22]). Commonly applied for the determination of the complex dielectric properties of very thin or strongly absorbing layers, generalized ellipsometry can also be used for measuring the birefringence of anisotropic samples ([23]). The investigation of birefringence by visible light is limited to optically transparent samples. For semi-transparent materials, so-called optical coherence tomography (OCT) was developed [24], allowing contactless depth-resolved imaging of internal structures using light in the near infrared (NIR). Although mainly used for biomedical applications, it has also found its way to the field of non-destructive testing, where numerous applications have been reported [25]. Its polarization-sensitive variant PS-OCT [26, 27] is capable of depth-resolved monitoring of birefringence in turbid samples [28, 29]. However, when materials are opaque for visible or NIR light, all these techniques fail, and the investigation of birefringence by contactless and non-destructive methods becomes a challenge. X-ray diffraction (XRD) can be employed for measuring the anisotropy of crystals, and even the birefringence of the crystalline phase can be deduced [30]. However, the use of ionizing radiation in general complicates its application. Thus, $\mathrm{THz}$ radiation seems to be highly promising for the investigation of birefringence in anisotropic samples due to its large penetration depth for many dielectric materials [31-33] and its non-ionizing properties.

\section{Birefringence at THz Frequencies}

Birefringence effects at $\mathrm{THz}$ frequencies have been reported for various materials. Different crystals, such as quartz [31], sapphire [31, 34], lithium niobate $\left(\mathrm{LiNbO}_{3}\right)$ [34-36], rutile [2], $\beta-\mathrm{BaB}_{2} \mathrm{O}_{4}$ (BBO) [37, 38], zinc oxide [39], the explosive 1,3,5-trinitro-S-triazine (RDX) [40], but also the organic crystal dimethyl amino 4-N-methylstilbazolium tosylate (DAST) 
[41] or molecular oxalic acid crystals [42] have shown to exhibit $\mathrm{THz}$ birefringence. For $\mathrm{LiNbO}_{3}$, very high values of $\Delta n$ up to 1.78 at $1 \mathrm{THz}$ were found [34], and even higher values of 3.3 at $1 \mathrm{THz}$ were observed for rutile [2]. For liquid crystals (LC), birefringence due to the alignment of the molecular chains was reported for pure materials [43-47] as well as for mixtures [45, 48-52], and also for a fiber-reinforced LC polymer component [6]. In this case, birefringence was also assigned to the orientation of the LC molecules and not to the alignment of the embedded fibers. The highest values of $\Delta n$ above 0.3 were found for the LC mixture 1852 [51] and for a fluorinated phenyl-tolane based nematic mixture [52]. For mechanically strained samples, only few studies on THz stress birefringence exist so far, such as for polytetrafluoroethylene (Teflon ${ }^{\circledR}$ ), where in addition the stress optical coefficient was measured [53], or for deformed or externally stressed polyethylene, where anisotropic material properties were proven $[54,55]$.

Furthermore, materials containing sub-wavelength structures were shown to exhibit $\mathrm{THz}$ birefringence. One highly topical field are metamaterials, where sub-wavelength metallic structures embedded in a dielectric material allow tailoring of dielectric properties. Strong birefringence in metamaterials has been simulated and/or experimentally proven for structures such as a cross-like geometry [56], split-ring and meander line designs [57], or for a cut wire pair [58]. Form birefringence was observed in alternatingly stacked structures of two different dielectric materials with sub-wavelength thickness, corresponding to onedimensional photonic crystals, such as two different polymers [59], silicon and air [60], but also for stacks of paper with air gaps in between [61]. Analogously, birefringence was reported for asymmetric hollow-core and polymer fibers [62, 63]. Furthermore, polymers reinforced by aligned glass fibers were found to be birefringent $[3,4,64]$ due to the embedded fibers representing sub-wavelength structures along one direction with regard to the relatively large $\mathrm{THz}$ wavelengths in the $100 \mu \mathrm{m}$ range. Moreover, $\mathrm{THz}$ birefringence was observed for textiles [53], a Yucca plant leaf [2], different types of wood [65, 66], but also for paper $[53,65,67]$, which exhibits a preferential orientation of the cellulose fibers due to the production process. In particular for wood it was demonstrated that the observed birefringence exhibits contributions not only from form birefringence but also from intrinsic birefringence due to the crystalline structure and microfibril angles of the wood fibers [66].

This large variety of examples emphasizes the significance of birefringence studies at $\mathrm{THz}$ frequencies. On the one hand, materials can be characterized with regard to their use for $\mathrm{THz}$ quasi-optical polarization devices. As an example, quartz plates [68], metamaterials [56, 58, 69], liquid crystals [70], but also paper [61] and wood [65] have been proposed and employed for the fabrication of monochromatic and, in the case of quartz, also achromatic $\mathrm{THz}$ wave plates. Moreover, especially when samples are opaque for visible or NIR light, valuable information about anisotropic material properties can be obtained, which are hard to get with other non-destructive methods. Besides birefringence (corresponding to the "magnitude" of anisotropy), the orientation of the optical axis (giving the preferential direction of anisotropy) is of special interest. Therefore, in the following chapters an overview of the experimental conditions for PS-THz-TDS required for the determination of birefringence will be given, and a closer look on the methods will be taken that allow an extraction of birefringence and the optical axis from the measured data.

\section{Polarization-Sensitive THz Measurements}

For the determination of $\mathrm{THz}$ birefringence based on polarization-sensitive TDS measurements, the resulting polarization state of a $\mathrm{THz}$ wave with known polarization is analyzed 
after probing the sample. Therefore, the generation of $\mathrm{THz}$ radiation with defined polarization and PS detection selective for a single polarization component of the $\mathrm{THz}$ wave is required. In nearly all studies reported in literature, linearly polarized $\mathrm{THz}$ waves were used for birefringence measurements. Thus, the techniques for $\mathrm{THz}$ generation and detection commonly applied in THz-TDS, such as optical rectification, electro-optical (EO) sampling or the use of photoconductive antennas (PCAs) (as discussed in more detail below), are perse well suited as they are intrinsically correlated with the emission and detection of a preferential polarization direction [71-74]. A current field of research is $\mathrm{THz}$ generation by two-color mixing in air plasmas using amplified femtosecond (fs) laser systems. It was demonstrated that the emitted $\mathrm{THz}$ pulses are linearly polarized, and their polarization can be continuously rotated by changing the relative phase of the two-color fields [75, 76]. With regard to PCAs, THz waves emitted by two-contact electrode structures, such as dipole-, stripline- or bow-tie geometries, exhibit a preferential polarization direction, but were shown also to have a slight cross-polarization component. For PCA dipole and bow-tie structures with attached substrate lens, an ellipticity of the emitted $\mathrm{THz}$ radiation was found in the range of a few percent of the main polarization component $[77,78]$. This property appeared to be a general characteristic of these lens-coupled systems [78]. When two-contact PCAs are used for PS detection, they are subject to the same restrictions as for $\mathrm{THz}$ generation. Although they predominantly detect a main polarization component, they also showed a frequency dependent sensitivity to cross-polarization, thus exhibiting a non-ideal polarization response $[79,80]$.

The characterization of the polarization state of a $\mathrm{THz}$ wave implies the knowledge of two (ideally orthogonal) polarization components. If only a single polarization component of the $\mathrm{THz}$ electric field is measured at once, the polarization components have to be recorded sequentially. In [81], a wire-grid polarizer was rotated after the sample to selectively measure the horizontal and vertical THz electric field components, respectively, not giving any details about the detection principle. Alternatively, the polarization sensitivity of the EO effect was directly exploited: by an appropriate rotation of the crystallographic axes of an EO (110) ZnTe detection crystal, only the desired polarization component produced a signal [82, 83]. A rotation of the detector can also be implemented with PCAs, where the use of fibercoupled antennas facilitates this procedure [84]. Nevertheless, these sequential measurements can be prone to alignment errors, are time-consuming and are therefore less suited, e.g., for applications where large areas are investigated. This problem was overcome in a recent work [85], where a technique based on the fast mechanical rotation of an EO (110) crystal and lock-in detection was introduced, allowing for a fast and precise determination of the polarization direction of a THz wave. Thus, rotating the EO detector becomes suitable also for polarization imaging applications, as the authors proved in [86], where the method was applied for topographic measurements with extremely high axial resolution.

Alternatively, a simultaneous detection of two polarization components seems to be advantageous, circumventing any potential problems of sequential measurements. One approach for simultaneously measuring two polarization components is to split the $\mathrm{THz}$ beam after probing the sample, and to measure the polarization components by two separate detectors, requiring an additional splitting of the fs-laser beam. This technique was already pursued in an early demonstration of PS-THz-TDS, using two independent PCAs oriented by $90^{\circ}$ to each other [87]. Also the use of two separate EO detection systems was reported in [88], however, no information on the orientation of the used ZnTe crystals was given. In both cases, a wire-grid was employed as a $\mathrm{THz}$ polarizing beam splitter for separation of the polarization components. In [5], a modified approach with a (111) ZnTe crystal was shown, where splitting of the $\mathrm{THz}$ beam was waived, and two orthogonal polarization components 
were detected sequentially by rotating a half wave plate in the path of the fs-probe beam. A simultaneous measurement was proposed by adding a second detection arm into the setup. A rather elegant approach for the simultaneous detection of different polarization components is the use of multi-contact PCAs, which comes without an additional splitting of the THzand the fs-laser beam. Different geometries of the electrodes were investigated, such as three-contact structures with orthogonal gaps $[89,90]$ or with tips arranged under an angle of $120^{\circ}$ [91], as well as four-contact antennas with a single gap [92]. When multi-contact antennas are used, artifacts such as a cross-polarization response between the gaps can influence the detected $\mathrm{THz}$ signal [4, 91, 92], and a careful alignment is necessary for obtaining a good PS-detection performance [4, 90].

In practice, metallic wire-grid $\mathrm{THz}$ polarizers with sub-wavelength period are widely used after the emitter or in front of the detector for cleaning up any cross-polarization components or for adjusting the polarization direction. It is noted that these wire-grid polarizers can influence the properties of the transmitted $\mathrm{THz}$ wave. First, their extinction ratios can be relatively low in the THz region (e.g., values of 8:1 for the electric field amplitude or about 60:1 for the power were reported in [93], or about 20:1 at $400 \mathrm{GHz}$ were found in [88]) compared to commercially available filters in the infrared or visible spectral range, thus not perfectly eliminating crosspolarization. Second, the wire-grids effect a first order time-differentiation of the s-polarization component parallel to the wires, i.e., in blocking direction, resembling a negative phase shift of the transmitted s-polarized leakage signal [94].

\section{Measurement of THz Birefringence}

\subsection{Known Orientation of the Optical Axis}

As discussed in Chap. 3, the investigation of birefringence in the THz spectral range is a wide field with numerous applications. In literature, in many cases birefringence was determined for samples with known orientation of the optical axis, such as for crystals [35, 36, 38], LCs [43-52] or form birefringent samples [59-61]. Theoretical considerations on how to choose appropriate Jones vectors for data analysis based on previous knowledge of the investigated sample and the detection geometry were presented in [95], underlined by experimental data for a birefringent quartz crystal. In practice, however, the ordinary and extraordinary rays can easily be measured and the respective refractive indices can directly be calculated from the time-domain signals if an adequate orientation of the linear polarization of the probing $\mathrm{THz}$ wave, the optical axis and the detection axis is chosen. In the simplest case, the polarization of the $\mathrm{THz}$ pulse and the single detection axis are parallel. The ordinary and extraordinary refractive indices are obtained from two separate measurements where the sample is rotated in between, once with the ordinary and the other time with the extraordinary axis of the sample aligned parallel to the polarization of the incident $\mathrm{THz}$ field. An example is shown in Fig. 2 [49], where a sample cell with the LC nematic mixture BL037 was measured for the two different orientations. In that case, the preferential direction of the sample was defined by the orientation of the molecular chains. From the time-domain data, the frequency dependent values of $n_{o}$ and $n_{e}$, and thus $\Delta n$ were directly calculated.

This procedure is straightforward and, in its simplicity, provides a valuable tool for measuring the birefringence of materials with known optical axis. However, its applicability is limited when samples with unknown orientation of the optical axis are investigated. 
a

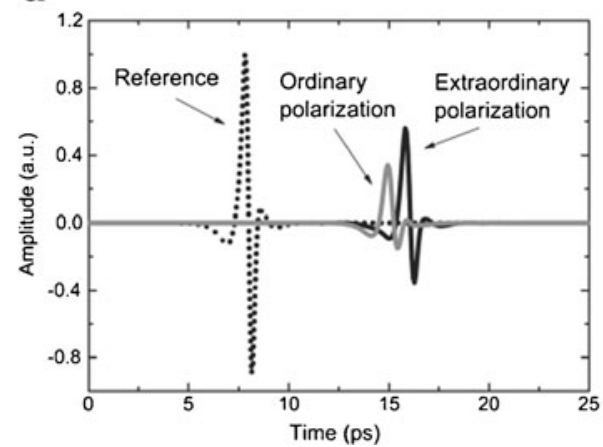

b

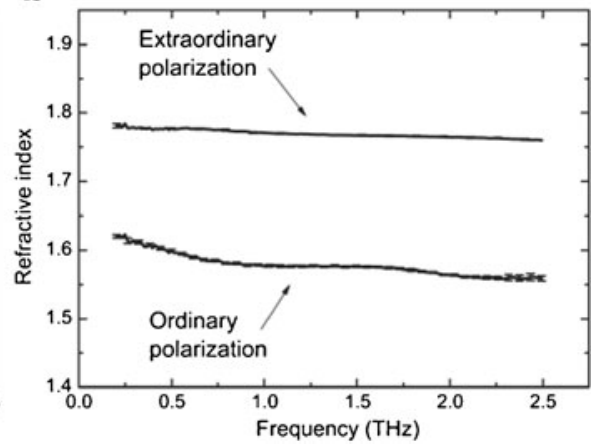

Fig. 2 PS-THz spectroscopy of the LC nematic mixture BL037. (a) Reference THz pulse and signals along ordinary and extraordinary axis. (b) Ordinary and extraordinary refractive indices, calculated from the data in (a) (Reprinted from [49], with permission from Elsevier)

\subsection{Determination of the Orientation of the Optical Axis}

As discussed above, knowledge of the orientation of the optical axis facilitates material analysis due to the simple measurement of $\Delta n$. Moreover, the optical axis itself is an interesting parameter for birefringent materials, as it provides information on the direction of anisotropy, such as the alignment of molecular chains or fibers, or the direction of stress inside a material. One frequently pursued approach for the determination of the optical axis is the measurement at different azimuthal angles by rotating the sample. Examples are given, e.g., in [34, 39-42], where the sample was rotated stepwise and a single polarization component was detected at each angular position. The extremes of the resulting time-domain curves as a function of the rotation angle allow an identification of the orientation of the optical axis, and the corresponding time-domain signals can be used for a direct evaluation of $\Delta n[34,39]$ analogous to Chap. 5.1. An example is shown in Fig. 3 [34], where the time-domain waveforms transmitted through a $\mathrm{LiNbO}_{3}$ crystal for varying azimuthal angle are shown.

Fig. 3 Transmitted THz time-domain waveforms measured for varying azimuthal angle for a $\mathrm{LiNbO}_{3}$ crystal (Reprinted from [34], with permission from The Optical Society OSA)

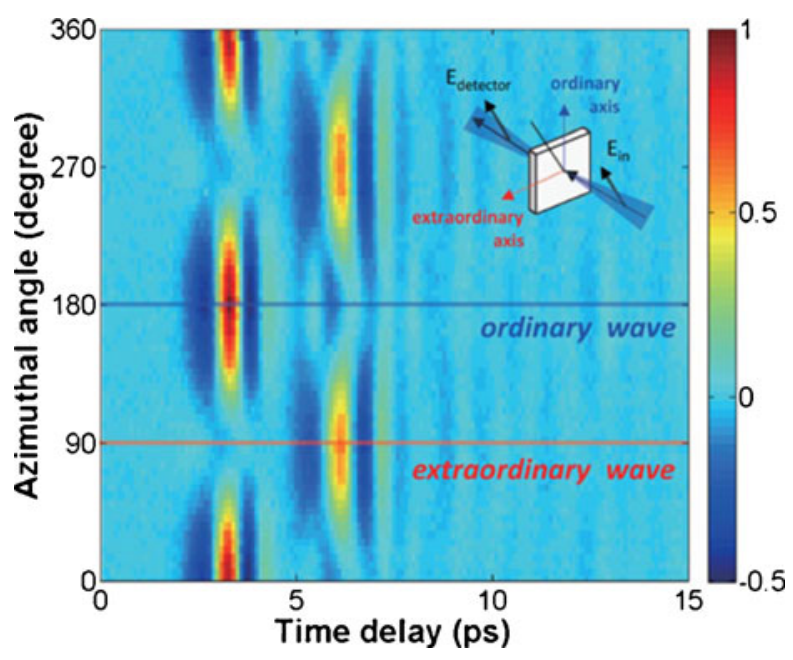


In [53], a modified arrangement with cross-polarized emitter and detector and an additional double-polarizer after the sample was chosen. The optical (principal) axis was determined as the azimuthal angle of the sample at which the cross-polarization component reduced to zero. It is noticeable that due to the use of double-polarizers exhibiting a higher extinction ratio than a single one, birefringence could be measured highly sensitive with smallest values of $\Delta n$ of $5^{*} 10^{-4}$. In [88], a simultaneous measurement of two orthogonal polarization components $E_{x}$ and $E_{y}$ was performed. The orientation of the optical axis of a $\mathrm{LiNbO}_{3}$ crystal was determined for a $45^{\circ}$ orientation of the $\mathrm{THz}$ emitter, where the ratio of $E_{y} / E_{x}$ evaluated at a single frequency exhibited maxima when the $\mathrm{THz}$ polarization coincided with the ordinary $(o)$ or extraordinary $(e)$ axis of the rotating sample. Also in [83], two orthogonal polarization components were detected, in that case by rotating a (110) ZnTe detection crystal by $90^{\circ}$. Maxima in the one and correspondingly minima in the other polarization component of the peak spectral amplitude as a function of the azimuthal angle were found for a quartz crystal sample when the $e$ - or $o$-axis was aligned along the detection axes. In addition, the authors presented equations based on Jones matrix formalism that allow extracting the direction of the optical axis, but also the phase retardation for an arbitrarily oriented sample. However, no further experimental evidence was given.

Rotating the sample can be tedious, especially when large and bulky samples are to be measured. A method that goes without rotation of the sample was introduced in [96], based on results of [97]. The presented system employed a two-color laser-induced gas plasma, where the orientation of the produced $\mathrm{THz}$ radiation was rotated by the relative phase of the two-color fields. In [97], a birefringent quartz crystal oriented by $45^{\circ}$ was inserted as an analyzer in front of an EO detector. A difference in the optical path lengths of the $o$ - and $e$ rays larger than the $\mathrm{THz}$ pulse width resulted in two separate peaks for the $o$ - and $e$ components of the time-domain signal. This arrangement was used to determine the orientation of the $\mathrm{THz}$ beam incident on the quartz crystal by the ratio of the time-domain maxima of the $e$ - and $o$-components when the $\mathrm{THz}$ polarization was rotated. In [96], the quartz analyzer was replaced by a birefringent sample with arbitrary orientation of the optical axis. In a first step, the ellipticity of the transmitted $\mathrm{THz}$ wave was determined as a function of frequency. Only frequencies where the $\mathrm{THz}$ wave was linearly polarized after the sample were considered for the subsequent extraction of the optical axis. This was done by rotating the polarization of the incident $\mathrm{THz}$ radiation, and the angles exhibiting minima of the measured $\mathrm{THz}$ signal were used for the extraction of the direction of the optical axis. Also here, the method was applicable only to samples with large birefringence leading to double pulses of the $e$ - and $o$-components. The described approaches were also extended for THz polarization imaging [9, 10], where texture anisotropies of SOFI (an insulation polyurethane foam material) were imaged. As no rotation of the sample is required, this method is less prone to errors due to re-alignment compared to the methods described at the beginning of the chapter. However, it requires an analysis of the ellipticity for selecting the frequencies that are relevant for the evaluation.

\subsection{Characterization of the THz Polarization State}

The birefringent properties of a sample can in principle be easily derived using suitable models based on, e.g., Jones (e.g., [98]) or Stokes vector formalism (e.g., [99]). For the latter the Mueller matrix contains all anisotropic information of the material under test (e.g., [100]). However, an application of these models for extracting the birefringence properties of the sample from the measuring data requires detailed knowledge of the polarization state of a $\mathrm{THz}$ wave, given by the main axes of the vibrational ellipsoid of the electric field and 
the phase difference between the polarization components. Exemplarily, a simple model using Jones formalism for a $\mathrm{THz}$ wave transmitted through a birefringent sample is given by the following equation:

$$
\left(\begin{array}{c}
\widetilde{E}_{x}(\omega) \\
\widetilde{E}_{y}(\omega)
\end{array}\right)=\widetilde{T}(\omega)^{*} R(\theta)^{*} \widetilde{M}(\omega)^{*} R(-\theta)^{*}\left(\begin{array}{c}
\widetilde{E}_{0 x}(\omega) \\
\widetilde{E}_{0 y}(\omega)
\end{array}\right)
$$

$\widetilde{E}_{0 x, 0 y}(\omega)$ and $\widetilde{E}_{x, y}(\omega)$ denote the complex THz signal in frequency space incident on and measured after transmission through the sample, respectively. For horizontally or vertically polarized $\mathrm{THz}$ radiation, the incident wave exhibits the simple directions $(1,0)$ or $(0,1)$, respectively. The determination of these parameters requires the phase-resolved measurement of the THz field components by THz-TDS. The matrix $\widetilde{T}(\omega)$ describes the detection properties of the THz system, which has to be determined by calibration measurements prior to the investigation of the sample. It includes the detection behavior of the $\mathrm{THz}$ detector as discussed, e.g., in $[80,91]$ and is assumed as complex in its general form. The unknown material parameters are comprised by the Jones matrices $R(\theta)$ and $\widetilde{M}(\omega)$

$$
R(\theta)=\left(\begin{array}{cc}
\cos \theta & -\sin \theta \\
\sin \theta & \cos \theta
\end{array}\right), \widetilde{M}(\omega)=\left(\begin{array}{cc}
e^{i \varphi_{0}(\omega)} & 0 \\
0 & e^{i \varphi_{0}(\omega)+\Delta \varphi(\omega)}
\end{array}\right) .
$$

$\widetilde{M}(\omega)$ describes the birefringent properties of the investigated material, i.e., a common phase shift $\varphi_{0}(\omega)$ and the difference in phase shift $\Delta \varphi(\omega)$ between the ordinary and extraordinary direction, representing the birefringence of the sample. $R(\theta)$ is the rotation matrix, giving the rotation $\theta$ of the optical axis with regard to the coordinate system of the measurement. Goal is the extraction of $\varphi_{0}(\omega), \Delta \varphi(\omega)$ and $\theta$ from the measuring data. From the above equations it is obvious that a more precise measurement of the polarization states of the incident and transmitted $\mathrm{THz}$ waves enables a more precise determination of the anisotropic material parameters of the sample.

Several examples where the full characterization of the polarization state of a $\mathrm{THz}$ wave was in the main focus of the work have been presented in literature. One is given in [101], where a polarimetry approach was introduced for the characterization of an arbitrary elliptically polarized $\mathrm{THz}$ wave, based on the use of a rotating polarizer and a fixed analyzer. The authors showed that three measurements at different orientations of the polarizer are required to determine the main axes of the vibrational ellipsoid and the phase shift, fully characterizing the polarization state of the $\mathrm{THz}$ wave. The method was also applied to measuring the frequency-dependent birefringence of a uniaxially birefringent crystal by orienting its optical axis by $45^{\circ}$ with regard to the horizontal. However, the results were ambiguous, as phase shifts of $\pm \pi / 4$ between the polarization components could not be distinguished, which was also a result of using only the measured electric field amplitudes in the calculations.

By exploiting also the phase information, this drawback was removed and an unambiguous characterization of the $\mathrm{THz}$ polarization state was obtained by calculating the four Stokes parameters in $[102,103]$. The authors used a single rotating polarizer in front of a two-contact PCA and derived the frequency-dependent Stokes parameters from measurements at three [102] or two [103] angles of the polarizer, respectively, where for the latter angles of $-45^{\circ}$ and $45^{\circ}$ where shown to be ideal. Moreover, in [102] an approach for 
measuring the Mueller matrix was proposed by rotating the sample and performing measurements at two azimuthal angles of the sample. A proof of principle was performed by determining the Mueller matrix of a $5 \mathrm{~mm}$ thick quartz crystal at different frequencies.

In a recent paper [104], a polarization modulation technique was presented for measuring the Jones vector of a $\mathrm{THz}$ wave. A fast rotating polarizer was used for modulating the polarization of the THz-pulse after transmission through a sample, and the vertical and horizontal electric field components were measured simultaneously by the in-phase and outof-phase channels, respectively, of a lock-in amplifier. In a detailed mathematical analysis of the problem in frequency space, also the non-idealities of a real setup, namely a possible rotation of the THz-polarization by off-axis parabolic mirrors and the finite extinction ratio of wire-gird polarizers were discussed. For sample characterization, one quantity of interest is the rotation angle of the THz polarization introduced by the sample. The authors showed that a very high precision better than $0.02^{\circ}$ was achieved for the measured rotation angle. In comparison, measurements with multi-contact $\mathrm{THz}$ antennas $[89,91]$ or static wire-grid polarizers [80] were capable of angular precisions of $0.2-0.3^{\circ}$. The presented approach was also used for determining the birefringence of a quartz crystal with the optical axis oriented by $45^{\circ}$ with regard to the horizontal. It was demonstrated that a single measurement with the polarization modulation technique yielded the same information as three measurements with static polarizers, in addition exhibiting improved accuracy and precision of the results. Altogether, the accurate measurement of the $\mathrm{THz}$ polarization state is still a challenging topic in the field of PS-THz-TDS, with promising results presented in the above studies.

\subsection{PS-THz Investigations of Fibrous Materials}

Looking at the methods for $\mathrm{THz}$ birefringent measurements reviewed in the previous chapters, well defined specimens such as quartz or other crystals were used for experimental evidence. The remainder of this paper will focus on the investigation of samples with less well defined anisotropic properties, namely fibrous materials that exhibit a preferential orientation of the fibers. As will be discussed below, the orientation of the optical axis (and also the degree of anisotropy) can vary over the sample. Consequently, the extraction of the anisotropic material properties becomes more sophisticated and requires specialized methods. In this context, especially the three works presented in [2-4] focusing on this topic will be reviewed in more detail. But first, some general considerations about fibrous materials will be given.

One important representative of fibrous materials are fiber reinforced polymers (RPs). They consist of a polymer matrix out of mainly thermosets or thermoplasts, in which glass, carbon or also natural fibers are embedded. The alignment of the fibers is intrinsic for the mechanical properties of the composite material, such as its tensile strength (e.g., $[105,106])$. For injection molded short-fiber RPs the fiber orientation and fiber orientation distribution is influenced, amongst others, by the flow pattern of the melt during the injection process - a parameter that in general can vary over the sample. The orientation of the fibers is commonly investigated destructively by microscopy of polished parts cut out of the sample. For glassfiber RPs, OCT (see Chap. 2) was shown to be suited for investigating the first few $100 \mu \mathrm{m}$ of the material [107], however, is limited for thicker samples. As the dimensions of the fibers with some tens of microns are much larger than the applied NIR wavelengths in the range of 800 to $1550 \mathrm{~nm}$, no form birefringence due to fiber alignment is observed with the polarizationsensitive variant PS-OCT. However, as the single fibers are resolved [107], OCT can be used as 
a kind of non-destructive depth-resolved microscopy for probing the orientation of the fibers in surface-near layers. In contrast, the measurement of birefringence by PS-THz-TDS is a potential tool for a non-destructive characterization of the fiber-orientation, as the dimensions of the fibers are smaller than the THz wavelengths. PS-THz-TDS works also for thicker samples, with high potential especially for glass and natural fibers. Oriented carbon fiber (CF) reinforced materials were shown to be highly anisotropic, since the fibers are electrically conducting while the epoxy matrix is not, with the penetration depth strongly depending on the orientation of the fibers with regard to the THz polarization [108-110]. However, their conductivity restricts the penetration depth of the THz waves. In [110], the skin depth of a unidirectional CFRP with a transverse conductivity of $10 \mathrm{~S} / \mathrm{m}$ was estimated to be as low as $200 \mu \mathrm{m}$ at $1 \mathrm{THz}$ and $500 \mu \mathrm{m}$ at $0.1 \mathrm{THz}$ for the electric field oriented normal to the fiber axis, i.e., for the orientation with the highest penetration depth. Thus, the applicability of PS-THz measurements to the birefringence analysis of CF reinforced materials is limited.

An early PS-THz study of fiber-reinforced materials was presented in [6], where a fiber-reinforced LC polymer was investigated. $\mathrm{THz}$ birefringence was evaluated at a selected sample position. By rotating the sample, the preferential orientation of the LC molecules and fibers, and consequently $\Delta n$ was determined. In addition, PS-THz imaging was performed, where a qualitative statement on the fiber orientation but no spatially resolved angular information was obtained. Furthermore, prove of birefringence was obtained by a crossed polarizer/analyzer arrangement. Although in this work no algorithm for a quantitative analysis was developed, clear evidence of the birefringence of the fiber-reinforced materials was given.

Later on, a quantitative analysis of the angular information of the fiber-reinforced LC polymer was conducted in [2]. Furthermore, the angular orientation of glass fibers in a RP material was investigated in [3]. For both studies, a standard transmission TDS-system was employed, where in addition a wire-grid polarizer between emitter and sample assured a perfectly polarized $\mathrm{THz}$ beam. Three different approaches for the extraction of the angular information of the fibers were performed, namely the calculation of the angular orientation from

1. the peak amplitudes of the transmitted $\mathrm{THz}$ signal

2. a least square approximation of the $\mathrm{THz}$ signal for a certain orientation angle

3. the refractive indices extracted for three different orientations of the sample.

The first approach (so called basic algorithm in [2]) is the simplest one and has some limitations, as it can only be applied to linear non-dichroistic samples with medium birefringence. Nevertheless, it is fast and easy to use, which is advantageous when working with imaging data. If a sample has a difference in the optical path lengths for the $o$ - and $e$ wave larger than the THz pulse width, it leads to two distinguishable pulse maxima. Then, the orientation angle $\theta$ of the incident $\mathrm{THz}$ pulse with regard to the optical axis can be calculated according to

$$
\theta=\operatorname{atan}\left(\sqrt{\frac{\widehat{\mathrm{e}}_{1}}{\widehat{\mathrm{e}}_{2}}}\right),
$$

where $\widehat{e}_{1}$ and $\widehat{e}_{2}$ are the peak amplitudes of the first and the second maximum, respectively, of the transmitted $\mathrm{THz}$ pulse corresponding to the $o$ - and $e$-wave.

The second approach (so called advanced algorithm in [2]) avoids the limitations of the first one. Yet, it requires either knowledge of the ordinary and extraordinary refractive 
indices or the separate measurement of both waves. The time-dependent THz signal $e(t)$ for a certain orientation angle $\theta$ can be written as

$$
e(t)=\sin ^{2}(\theta) \cdot e_{o}(t)+\cos ^{2}(\theta) \cdot e_{e}(t),
$$

with

$$
\begin{aligned}
& e_{o}(t)=e_{r e f}(t) * h_{o}(t) \\
& e_{e}(t)=e_{r e f}(t) * h_{e}(t) .
\end{aligned}
$$

Here, $e_{r e f}(t)$ and $h(t)$ denote the reference pulse and the pulse response of the sample, respectively. A numerical optimization is performed for $e_{\text {error }}(t)$

$$
e_{\text {error }}(t):=e_{\text {sim }}(t)-e_{\text {meas }}(t),
$$

which gives the orientation angle $\theta$ from a comparison of the calculated ( $\mathrm{sim}$ ) and measured (meas) THz signal.

In [2], results of this algorithm were presented for different sample materials. The most interesting one is a fiber-reinforced LC polymer, which is used in a part of a helicopter stator blade. A detailed description of the material can be found in [2]. Figure 4 shows the results of the orientation analysis. In (a), the basic approach was employed for $\mathrm{THz}$ imaging data, whereas in (b) the advanced approach was used to determine the angular orientation for the marked positions in the photo. Both results are based on time-domain data and are therefore not assigned to a specific $\mathrm{THz}$ frequency. The observed angles cover a range between $20^{\circ}$ and $70^{\circ}$, and the results of both approaches agree well.

The third approach presented in [3] does not require the knowledge or measurement of the $o$ - and $e$-refractive indices as necessary for the second one. Therefore, it is more flexible to apply, however, three consecutive measurements at three different orientation angles of the sample have to be made. Typically, the best results are obtained for angles of $0^{\circ}, 45^{\circ}$ and $90^{\circ}$, leading to three corresponding refractive indices $n_{0^{\circ}}, n_{45^{\circ}}$ and $n_{90^{\circ}}$. These refractive indices are effective parameters to evaluate the birefringence of the sample under test; the

a

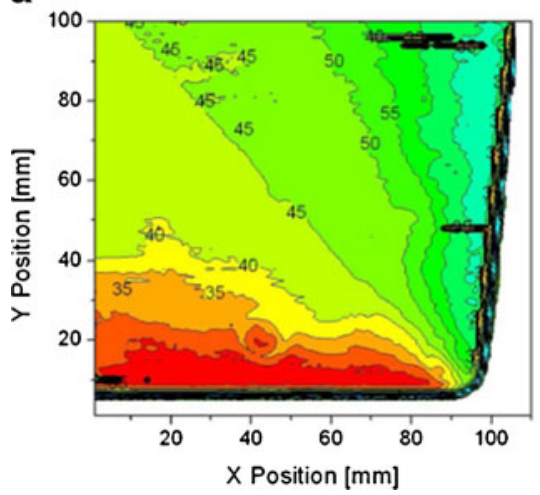

b

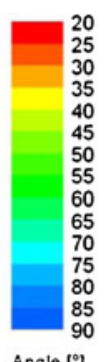

Angle [']

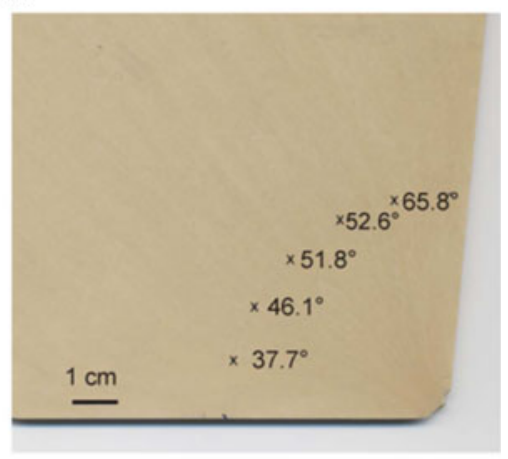

Fig. 4 Orientation analysis based on time-domain data for a fiber-reinforced LC polymer: (a) THz image with orientations obtained from basic approach and (b) photograph, where the numbers with crosses indicate the orientations obtained from the advanced approach. (Reprinted from [2], with permission from The Optical Society OSA) 
physically meaningful quantities are $n_{o}$ and $n_{e}$. Each refractive index can be written as a function of the ordinary and extraordinary refractive index, considering an index ellipsoid similar to the one in Fig. 1:

$$
\begin{aligned}
n_{0^{\circ}} & =n_{e} \cdot \cos ^{2}(\theta)+n_{o} \cdot \sin ^{2}(\theta) \\
n_{45^{\circ}} & =n_{e} \cdot \cos ^{2}\left(\theta+45^{\circ}\right)+n_{o} \cdot \sin ^{2}\left(\theta+45^{\circ}\right) \\
n_{90^{\circ}} & =n_{e} \cdot \cos ^{2}\left(\theta+90^{\circ}\right)+n_{o} \cdot \sin ^{2}\left(\theta+90^{\circ}\right) .
\end{aligned}
$$

From these three equations, the unknown parameters $n_{e}, n_{o}$ and the orientation angle $\theta$ can be derived. Furthermore, also the additive content as well as the degree of fiber orientation in a glass fiber-reinforced plastic was calculated according to the model of Polder and van Santen for the effective permittivity. For further details see [3].

The described algorithm was applied to a polyamide flat plate sample containing $30 \mathrm{wt} . \%$ of glass fibers. Figure 5 (a) shows the obtained fiber orientation angle $\theta$ as well as the fraction of oriented fibers indicated by the direction and length of the arrows, respectively, where the refractive index at $0.6 \mathrm{THz}$ was used to determine these parameters. Note that orientation angle and fraction of orientation are the resulting average across the sample thickness. Figure 5 (b) and (c) depict results from a Moldflow ${ }^{\circledR}$ simulation for the $x y$-plane and the $y z$-plane of the sample. Altogether, a good agreement between measurement and simulation is found. The fibers are mainly orientated along the mold flow with some larger deviation for point 1 at the end of the sample (due to the end of the mold flow). The fraction of orientated fibers is higher for the points 4-7 compared to 1-3, which was attributed to the occurrence of a middle layer with up to $20 \%$ of fibers with an orientation tensor in $x$ direction (see Fig. 5 (c)). Such a layer is less pronounced in the outer rim of the sample leading to a larger fraction of orientated fibers at those points. For a detailed discussion of the results see [3].
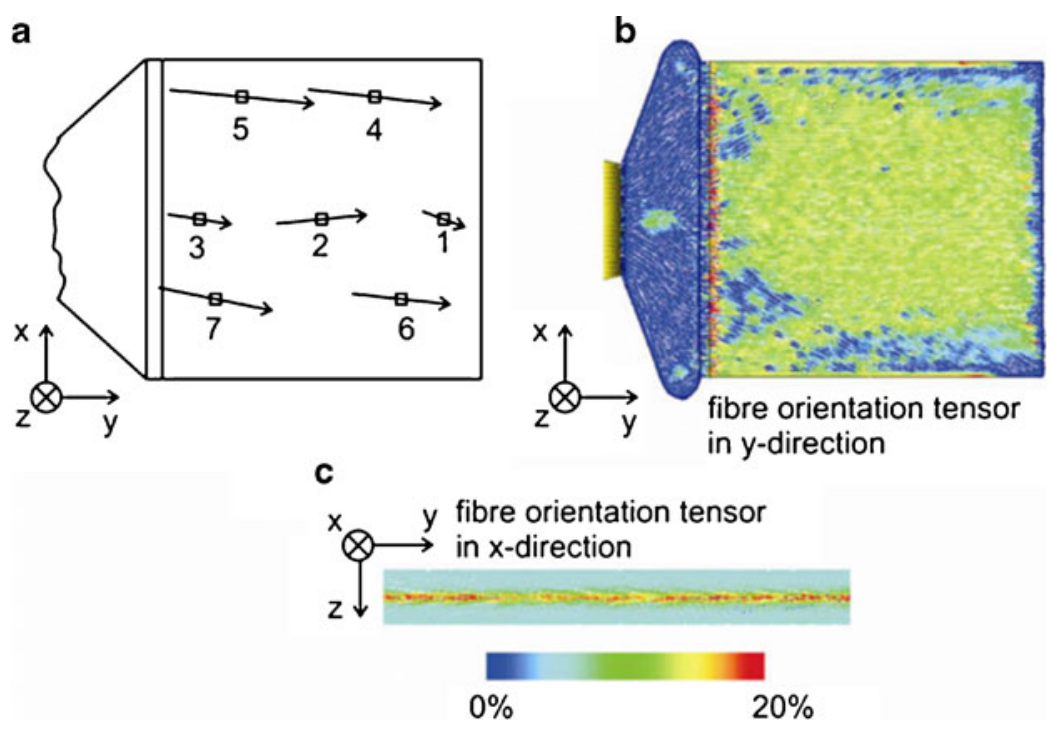

Fig. 5 (a) Schematic of flat plate sample with preferential orientations, extracted at $0.6 \mathrm{THz}$. Simulation results: amount of fibers orientated in (b) $y$-direction and (c) $x$-direction (Reprinted from [3], with permission from Elsevier) 
One limitation of the presented approach should be noted: if birefringence becomes larger or the sample thicker such that two separate peaks for ordinary and extraordinary wave are observed in the $\mathrm{THz}$ signal, the data extraction algorithm will not give reasonable values for $n_{0^{\circ}}, n_{45^{\circ}}$ and $n_{90^{\circ}}$. Therefore, the algorithm would require further adaption when higher birefringent samples are under investigation.

A completely different approach compared to the results presented in literature was pursued in [4] and was thereafter applied to the investigation of glass-fiber RP samples. Here, a concept originally developed for PS-OCT (as described, e.g., in [29]) was adopted and applied to $\mathrm{THz}$ birefringence measurements and $\mathrm{PS}-\mathrm{THz}$ imaging. The system itself is based on a scanning THz-TDS system in reflection geometry developed in [111], where the $\mathrm{THz}$ beam is scanned by an $x y$-galvanometer scanner through a scanning lens. A schematic of the PS-THz imaging system is shown in Fig. 6. In contrast to most other $\mathrm{THz}$ birefringence measurements, the sample is irradiated by a quasi-circularly polarized THz-pulse, which is produced by total internal reflection of linearly polarized $\mathrm{THz}$ radiation in a high-resistivity silicon Fresnel prism, which acts as an achromatic quarter wave plate [112]. After reflection by the sample, the $\mathrm{THz}$ pulse passes the prism a second time and is collected by a PS-detector for simultaneously measuring two polarization components. The main idea is simple: in the case of a non-birefringent sample, again linear polarization is obtained after the prism; a birefringent sample in turn results in a generally elliptically polarized wave. However, extracting the birefringence and optical axis of the material from the measuring data requires a detailed mathematical analysis of the situation.

Based on Jones matrix formalism, the authors showed that the problem can be reduced to relatively simple equations. For the first time, an algorithm was presented where evaluation was not performed at single frequencies, but which exploits the frequency dependence of the $\mathrm{THz}$ measurement for extracting the $\mathrm{THz}$ birefringent parameters. In detail, the complex frequency dependent spectral components of the two polarization components of the signal reflected from the rear sample surface, $S_{a}$

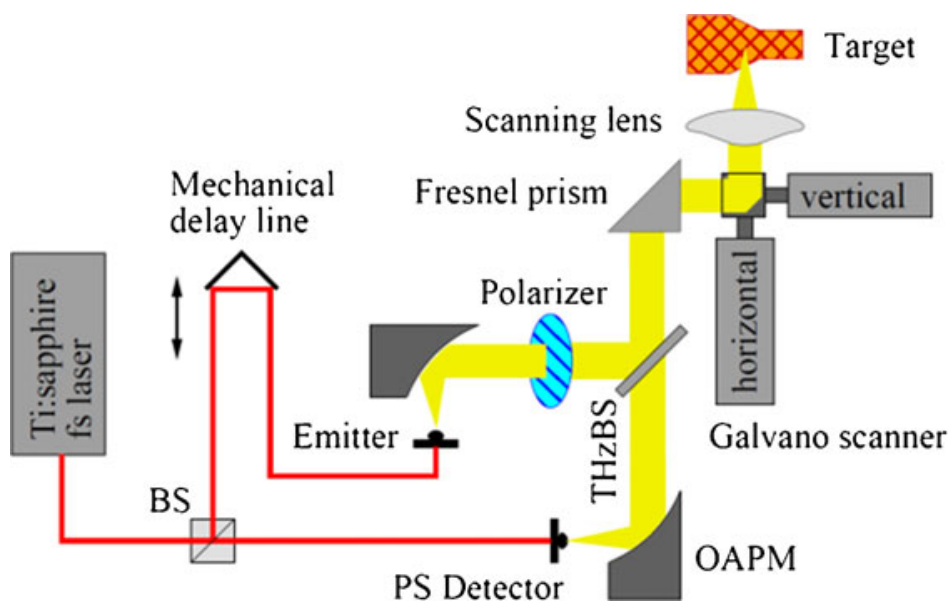

Fig. 6 Schematic drawing of the PS-THz imaging system. BS: beam splitter, OAPM: off-axis parabolic mirror (Reprinted from [4], with permission from The Optical Society OSA) 
and $S_{b}$, were used for calculation. The parameter $A^{2}$ used for normalization was defined as the spectral energy of the signal. A minimum at a frequency $f_{\min }$ of the relation

$$
g_{1}(f)=\frac{\left|S_{a}+S_{b}\right|}{A}
$$

was shown to occur at a phase shift equal to $\pi$ between the two polarization components. Correlating the phase shift $\Delta \varphi$ with $\Delta n$ (see Chap. 2), the average birefringence in a point of the sample with known thickness was obtained. Furthermore, the relation

$$
g_{2}(f)=\frac{\left|S_{a}\right|^{2}-\left|S_{b}\right|^{2}}{2 A^{2}}
$$

was shown to exhibit an amplitude equal to $\sin (2 \theta)$, where $\theta$ is the orientation of the optical axis with regard to the horizontal, allowing to extract the orientation of the optical axis from the measurements.

Several points of this method are worth mentioning: first, it requires only a single time-domain depth scan with a PS-detector, as it relies on the relative signals between the two polarization components only. Thus, there is no need of recording a reference or measuring at different rotation angles. Therefore, this approach is well suited for imaging, but can evidently also be used for fast single-point measurements. Second, a three-contact PCA similar to the one in [89] was used for PS-detection. It was shown that the algorithm requires a symmetric adjustment of the detection arms, but otherwise is not sensitive to the degree of cross-talk between the gaps, resulting in more or less non-orthogonal effective detection axes. Furthermore, the inverse of the minimum detectable birefringence depends on the bandwidth times the sample thickness, i.e., thinner samples will require $\mathrm{THz}$ systems with higher bandwidths for measuring the same birefringence as thicker samples. The measurements in reflection geometry double the optical path length and therefore the minimum detectable value of $\Delta n$. It is noted that the algorithm does not yield frequency resolved values of the birefringence and works only well for linear non-dichroitic samples with negligible dispersion. However, considering these limitations, the algorithm can deliver efficient and fast information on the birefringence and orientation of the optical axis of a sample.

The algorithm was tested for the spatially resolved investigation of a short-fiber injection molded polymer test bar (high-density polyethylene with $50 \mathrm{wt} . \%$ of glass-fibers), shown in Fig. 7 (a). The extracted orientation of the optical axis and the values of birefringence are indicated by the direction of the arrows and the color-coding (color online), respectively, in Fig. 7 (b).

The orientation of the optical axis is assumed to correlate with the average fiber alignment, resulting from the flow pattern during injection molding. The reduced birefringence in the left, wider part is assigned to distortions in the fiber alignment possibly due to turbulences when the melt was injected from the left. Altogether, this example shows that complete information on the birefringence properties of the RP sample and of other anisotropic materials in general can be obtained by the presented setup and algorithm, relying only on a single measurement with a PS-detector. 

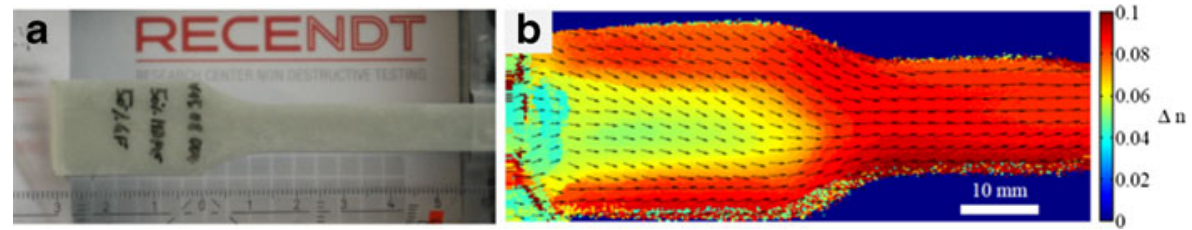

Fig. 7 (a) Injection molded glass-fiber RP test bar. (b) Orientation of the optical axis (arrows) and birefringence $\Delta n$, determined from the frequency dependence of the THz signals (color-coded; color online) (Reprinted from [4], with permission from The Optical Society OSA)

\section{Conclusion and Outlook}

In this paper, recent results on $\mathrm{THz}$ birefringence measurements were reviewed. A basic introduction to birefringence, an overview over different samples birefringent at $\mathrm{THz}$ frequencies, and a short look at the technical requirements for PS-THz measurements were given. Moreover, different approaches for $\mathrm{THz}$ birefringence measurements presented in literature were discussed. It was pointed out that it explicitly has to be distinguished between the situations where samples with known or unknown orientation of the optical axis are investigated, where the latter represents the more challenging situation with regard to the extraction of birefringence. Different approaches were reviewed for the identification of the optical axis of a material, employing a rotation of the sample or of the THz polarization, and requiring different numbers of measurements. In addition, works on the full characterization of $\mathrm{THz}$ polarization were discussed.

The last part of the paper was focusing on methods for the analysis of anisotropic fibrous materials, where the orientation of the optical axis as well as birefringence are relevant parameters, as the earlier corresponds to the average fiber orientation, whereas the latter can be seen as the degree of anisotropy, being influenced by the degree of fiber alignment as well as by the fiber concentration [3]. Therefore, the measurement of the optical axis as well as of birefringence is also of practical importance, e.g., for quality control of fiber RP materials. Three specific works [2-4] were reviewed in detail, where different approaches based on PSTHz-TDS for the analysis of fibrous materials were presented. In practice, a method clearly favorable does not require sample rotation and relies only on a minimum number of measurements - a requirement that was met by the approach presented in [4].

Although the recent works done in the field of $\mathrm{THz}$ birefringence measurements have demonstrated the high potential of PS-THz technology for the investigation of optically opaque anisotropic samples, still further developments are necessary. The requirements for a future use of PS-THz technology in non-destructive testing and quality control can be summarized by two main points: the efficient determination of the (unknown) orientation of the optical axis, together with the measurement of birefringence. Besides developing of appropriate and, especially with regard to imaging, efficient PS-THz approaches, such as by irradiation with quasi-circularly polarized $\mathrm{THz}$ radiation [4] or the fast rotation of detectors [85], the establishment of models and algorithms for parameter extraction, e.g., based on Jones or Stokes vector formalisms, is intrinsic. What currently still complicates modeling of data and limits the sensitivity of PS-THz measurements are the non-ideal properties of many components used in PS-THz technology, such as of polarizers with low extinction ratio and PS-detectors with non-ideal detection properties. An example are multi-contact PCAs, where cross-talk between the detected polarization components and the high sensitivity with regard to alignment heavily influence the measured signal. Thus, one focus should be the 
development of high-quality PS-THz components, including broadband polarizers with high extinction ratio or reliable PS-THz detectors. Moreover, other standard components available in other wavelength ranges, such as broadband waveplates, are not or hardly available at $\mathrm{THz}$ frequencies $[68,112]$ and therefore limit the development of new techniques. In our opinion, the development of new and improved broadband PS-THz components is a highly important issue, which could bring $\mathrm{THz}$ birefringence measurements to its next level.

Acknowledgments This work was supported by the European Regional Development Fund (EFRE) in the framework of the EU-programme Regio 13, and the federal state Upper Austria. Further funding was received by the "K-Project for Non-Destructive Testing and Tomography" supported by the COMET-program of the Austrian Research Promotion Agency (FFG). Part of this work was supported by the Austrian Science Fund FWF (Project L507-N20). Thanks go to the THz group at RECENDT, especially to Stefan Katletz and Michael Pfleger for their work on PS-THz technology, and to the THz-group of Martin Koch at the PhilippsUniversity Marburg for their support and fruitful cooperation.

Open Access This article is distributed under the terms of the Creative Commons Attribution License which permits any use, distribution, and reproduction in any medium, provided the original author(s) and the source are credited.

\section{References}

1. P. U. Jepsen, D. G. Cooke, M. Koch, Laser\&Photonics Rev. 5, 124 (2011)

2. C. Jördens, M. Scheller, M. Wichmann, M. Mikulics, K. Wiesauer, M. Koch, Appl. Optics 48, 2037 (2009)

3. C. Jördens, M. Scheller, S. Wietzke, D. Romeike, C. Jansen, T. Zentgraf, K. Wiesauer, V. Reisecker, M. Koch, Comp. Sci. Technol. 70, 472 (2010)

4. S. Katletz, M. Pfleger, H. Pühringer, M. Mikulics, N. Vieweg, O. Peters, B. Scherger, M. Scheller, M. Koch, K. Wiesauer, Opt. Express 20, 23025 (2012)

5. N. C. J. van der Valk, W. A. M. van der Marel, P. C. M. Planken, Opt. Lett. 30, 2802 (2005)

6. F. Rutz, T. Hasek, M. Koch, H. Richter, U. Ewert, Appl. Phys. Lett. 89, 221911 (2006)

7. B. Gompf, N. Gebert, H. Heer, M. Dressel, Appl. Phys. Lett. 90, 082104 (2007)

8. X. Wang, Y. Cui, J. S. Je, Y. Zhang, J. Opt. Soc. Am. A 27, 2387 (2010)

9. L. Zhang, H. Zhong, C. Deng, C. Zhang, Y. Zhao, Opt. Commun. 283, 4993 (2010)

10. L. Zhang, H. Zhong, C. Deng, C. Zhang, Y. Zhao, Opt. Commun. 284, 4356 (2011)

11. T. Nagashima, M. Hangyo, Appl. Phys. Lett. 79, 3917 (2001)

12. N. Matsumoto, T. Fujii, K. Kageyama, H. Takagi, T. Nagashima, M. Hangyo, Jpn. J. Appl. Phys. 48, 09KC11 (2009)

13. N. Matsumoto, T. Hosokura, T. Nagashima, M. Hangyo, Opt. Lett. 36, 265 (2011)

14. M. Neshat, N. P. Armitage, Opt. Express 20, 29063 (2012)

15. R. Bartholin, Experimenta Crystalli Islandici Disdiaclastici Quibus Mira \& Insolita Refractio Detegitur (D. Paulli, Copenhagen, 1669), pp. 60

16. M. Born, E. Wolf, Principles of Optics, 7th edn. (Cambridge University Press, Cambridge, 1999), pp. 234

17. F. Pockels, Lehrbuch der Kristalloptik (1906)

18. J. Kerr, Philosophical Magazine Series 4 50, 337 (1875)

19. I.-C. Khoo, Liquid Crystals, 2nd edn. (John Wiley \& Sons Inc., New Jersey, 2007)

20. M. Born, E. Wolf, Principles of Optics, 7th edn. (Cambridge University Press, Cambridge, 1999), pp. 837

21. K. Ramesh, Digital Photoelasticity, 1st edn. (Springer, 2000)

22. H. Fujiwara, Spectroscopic Ellipsometry, John Wiley \& Sons Inc., Chichester 2008, pp. 209

23. G. E. Jellison Jr., Thin Solid Films 450, 42 (2004)

24. D. Huang, E. A. Swanson, C. P. Lin, J. S. Schuman, W. G. Stinson, W. Chang, M. R. Hee, T. Flotte, K. Gregory, C. A. Puliafito, J. G. Fujimoto, Science 254, 1178 (1991)

25. D. Stifter, Appl. Phys. B 88, 337 (2007)

26. R. Hee, D. Huang, E. A. Swanson, J. G. Fujimoto, J. Opt. Soc. Am B 9, 903 (1992)

27. J. F. de Boer, T. E. Milner, M. J. C. van Gemert, J. S. Nelson, Opt. Lett. 22, 934 (1997)

28. D. Stifter, P. Burgholzer, O. Höglinger, E. Götzinger, C. K. Hitzenberger, Appl. Phys. A 76, 947 (2003) 
29. K. Wiesauer, M. Pircher, E. Götzinger, C. K. Hitzenberger, R. Engelke, G. Ahrens, G. Grützner, D. Stifter, Opt. Express 14, 5945 (2006)

30. J. J. Senkevich, S. B. Desu, V. Simkovic, Polymer 41, 2379 (2000)

31. D. Grischkowsky, S. Keiding, M. van Exeter, C. Fattinger, J. Opt. Soc. Am. B 7, 2006 (1990)

32. G. Zhao, M. ter Mors, T. Wenckenbach, P. C. M. Planken, J. Opt. Soc. Am. B 19, 1476 (2002)

33. R. Piesiewicz, C. Jansen, S. Wietzke, D. Mittleman, M. Koch, T. Kürner, Int. J. Infrared Milli. Waves 28, 363 (2007)

34. Y. Kim, M. Yi, B. G. Kim, J. Ahn, Appl. Optics 50, 2906 (2011)

35. S. Yi-Min, M. Zong-Liang, H. Bi-Hui, L. Guo-Qing Wang Li, Chin. Phys. Lett 24, 414 (2007)

36. D. Li, G. Ma, J. Ge, S. Hu, N. Dai, Appl. Phys. B 94, 623 (2009)

37. E. Estacio, S. Saito, T. Nakazato, Y. Furukawa, N. Sarukura, M. Cadatal, M. Hong Pham, C. Ponseca Jr., H. Mizuseki, Y. Kawazoe, Appl. Phys. Lett. 92, 091116 (2008)

38. J. Liu, X. Guo, J. Dai, X.-C. Zhang, Appl. Phys. Lett. 93, 171102 (2008)

39. Y. Kim, J. Ahn, B. G. Kim, D.-S. Yee, Jpn. J. Appl. Phys. 50, 030203 (2011)

40. V. H. Whitley, D. E. Hooks, K. J. Ramos, J. F. O’Hara, A. K. Azad, A. J. Taylor, J. Barber, R. D. Averitt, Anal. Bioanal. Chem. 395, 315 (2009)

41. X.-C. Zhang, X. F. Ma, Y. Jin, T.-M. Lu, Appl. Phys. Lett. 61, 3080 (1992)

42. R. Singh, D. K. George, A. Markelz, Proc. of the 36th International Conference on Infrared, Millimeter and Terahertz Waves (IRMMW-THz), pp. 1-2 (2011)

43. N. Vieweg, M. K. Shakfa, B. Scherger, M. Mikulics, M. Koch, J. Infrared Milli. Terahz. Waves 31, 1312 (2010)

44. N. Vieweg, C. Jansen, M. K. Shakfa, M. Scheller, N. Krumbholz, R. Wilk, M. Mikulics, M. Koch, Opt. Express 18, 6097 (2010)

45. N. Vieweg, M. Koch, Appl. Opt. 49, 5764 (2010)

46. N. Vieweg, M. K. Shakfa, M. Koch, J. Infrared Milli. Terahz. Waves 32, 21 (2011)

47. R. Wilk, N. Vieweg, O. Kopschinski, T. Hasek, M. Koch, J. Infrared Milli. Terahz. Waves 30, 1139 (2009)

48. C. S. Yang, C. J. Lin, R. P. Pan, C. T. Que, K. Yamamoto, M. Tani, C. L. Pan, J. Opt. Soc. Am. B 27, 1866 (2010)

49. N. Vieweg, M. K. Shakfa, M. Koch, Opt. Commun. 284, 1887 (2011)

50. H. Park, E. P. J. Parrott, F. Fan, M. Lim, H. Han, V. G. Chigrinov, E. Pickwell-MacPherson, Opt. Express 20, 11899 (2012)

51. M. Reuter, K. Altmann, N. Vieweg, K. Garbat, R. Dabrowski, M. Koch, Proc. of the 7th German Microwave Conference (GeMiC), pp. 1 - 3 (2012)

52. L. Wang, X.-W. Lin, X. Liang, J.-B. Wu, W. Hu, Z.-G. Zheng, B.-B. Jin, Y.-Q. Qin, Y.-Q. Lu, Opt. Mater. Express 2, 1314 (2012)

53. S.-I. Ebara, Y. Hirota, M. Tani, M. Hangyo, Proc. of the Joint 32nd International Conference on Infrared and Millimeter Waves and the 15th International Conference on Terahertz Electronics (IRMMW-THz), pp. $666-667$ (2007)

54. T. Tanabe, K. Watanabe, Y. Oyama, K. Seo, NDT\&E Internat. 43, 329 (2010)

55. N. Fuse, T. Takahashi, Y. Ohki, R. Sato, M. Mizuno, K. Fukunaga, IEEE Electr. Insul. M. 27, 26 (2011)

56. C. Imhof, R. Zengerle, Opt. Commun. 280, 213 (2007)

57. A. C. Strikwerda, K. Fan, H. Tao, D. V. Pilon, X. Zhang, R. D. Averitt, Opt. Express 17, 136 (2009)

58. P. Weis, O. Paul, C. Imhof, R. Beigang, M. Rahm, Appl. Phys. Lett. 95, 171104 (2009)

59. M. Scheller, C. Jördens, M. Koch, Opt. Express 18, 10137 (2010)

60. S. C. Saha, Y. Ma, J. P. Grant, A. Khalid, D. R. S. Cumming, IEEE Photon. Technol. Lett. 22, 79 (2010)

61. B. Scherger, M. Scheller, N. Vieweg, S. T. Cundiff, M. Koch, Opt. Express 19, 24884 (2010)

62. S. Atakaramians, S. Afshar Vahid., B. M. Fischer, D. Abbott, T. M. Monro, Opt. Commun. 282, 36 (2009)

63. M. Cho, J. Kim, H. Park, Y. Han, K. Moon, E. Jung, H. Han, Opt. Express 16, 7 (2008)

64. F. Rutz, S. Wietzke, M. Koch, H. Richter, S. Hickmann, V. Trappe, U. Ewert, Proc. of the European Conference on Non-destructive Testing (ECNDT), paper We2.8.2 (2006)

65. M. Reid, R. Fedosejevs, Appl. Opt. 45, 2766 (2006)

66. T. M. Todoruk, Ian D. Hartley, Matthew E. Reid, IEEE Transact. THz Sci. Technol. 2,123 (2012)

67. T. Hattori, H. Kumon, H. Tamazumi, Proc. of the 35 th International Conference on Infrared, Millimeter and Terahertz Waves (IRMMW-THz), pp. 1-2 (2010)

68. J.-B. Masson, G. Gallot, Opt. Lett. 31, 265 (2006)

69. X. G. Peralta, E. I. Smirnova, A. K. Azad, H.-T. Chen, A. J. Taylor, I. Brener, J. F. O’Hara, Opt. Express 17,773 (2009)

70. C.-F. Hsieh, R.-P. Pan, T.-T. Tang, H.-L. Chen, C.-L. Pan, Opt. Lett. 31, 1112 (2006) 
71. A. Rice, Y. Jin, X. F. Ma, X.-C. Zhang, Appl. Phys. Lett. 64, 1324 (1994)

72. Q. Chen, X.-C. Zhang, Appl. Phys. Lett. 74, 3435 (1999)

73. R. Shimano, H. Nishimura, T. Sato, Jpn. J. Appl. Phys. 44, L 676 (2005)

74. P. C. M. Planken, H.-K. Nienhuys, H. J. Bakker, T. Wenckebach, J. Opt. Soc. Am. B 13, 313 (2001)

75. H. Wen, A. M. Lindenberg, Phys. Rev. Lett. 103, 023902 (2009)

76. J. Dai, N. Karpowicz, X.-C. Zhang, Phys. Rev. Lett. 103, 023001 (2009)

77. J. Van Rudd, J. L. Johnson, D. M. Mittleman, Opt. Lett. 20, 1556 (2000)

78. J. Van Rudd, J. L. Johnson, D. M. Mittleman, J. Opt. Soc. Am. B 18, 1524 (2001)

79. Y. Gong, H. Dong, Z. Chen, Terahertz Science and Technology 4, 137 (2011)

80. M. Neshat, N. P. Armitage, Opt. Lett. 37, 1811 (2012)

81. Y. Ikebe, T. Morimoto, R. Masutomi, T. Okamoto, H. Aoki, R. Shimano, Phys. Rev. Lett. 104, 256802 (2010)

82. J. N. Heyman, D. Bell, T. Khumalo, Appl. Phys. Lett. 88, 162104 (2006)

83. L.-L. Zhang, H. Zhong, C. Deng, C.-L. Zhang, Y.-J. Zhao, Appl. Phys. Lett. 94, 211106 (2009)

84. B. Scherger, N. Born, C. Jansen, S. Schumann, M. Koch, K. Wiesauer, IEEE Transactions on Terahertz Science and Technology 2, 556 (2012)

85. N. Yasumatsu and S. Watanabe, Rev. Sci. Instrum. 83, 023104 (2012)

86. N. Yasumatsu and S. Watanabe, Opt. Lett. 37, 2706 (2012)

87. D. M. Mittleman, J. Cunningham, M. C. Nuss, M. Geva, Appl. Phys. Lett. 71, 26 (1997)

88. M. B. Byrne, M. U. Shaukat, J. E. Cunningham, E. H. Linfield, A. G. Davies, Appl. Phys, Lett. 98, $151104(2011)$

89. E. Castro-Camus, J. Lloyd-Hughes, M. B. Johnston, M. D. Fraser, H. H. Tan, C. Jagadish, Appl. Phys. Lett. 86, $254102(2005)$

90. E. Castro-Camus, J. Lloyd-Hughes, L. Fu, H.H. Tan, C. Jagadish, M. B. Johnston, Opt. Express 15, 7047 (2007)

91. H. Makabe, Y Hirota, M Tani, M Hangyo, Opt. Express 15, 11650 (2007)

92. A. Hussain, S. R. Andrews, Opt. Express 16, 7251 (2008)

93. E. Castro-Camus, J. Infrared Milli. Terahz. Waves 33, 418 (2012)

94. A. Filin, M. Stowe, R. Kersting, Opt. Lett. 26, 2008 (2001)

95. E. Castro-Camus, M. B. Johnston, Opt. A: Pure Appl. Opt. 11, 105206 (2009)

96. L. Zhang, H. Zhong, C. Deng, C. Zhang, Y. Zhao, Opt. Express 18, 20491 (2010)

97. L. Zhang, H. Zhong, C. Deng, C. Zhang, Y. Zhao, Opt. Express 17, 20266 (2009)

98. K. K. Sharma, Optics - Principles and Applications (Elsevier, Burlington San Diego London, 2006) pp. 129

99. K. K. Sharma, Optics - Principles and Applications (Elsevier, Burlington San Diego London, 2006) pp. 139

100. K. K. Sharma, Optics - Principles and Applications (Elsevier, Burlington San Diego London, 2006) pp. 150

101. J.-B. Masson, G. Gallot, Conference on Lasers \& Electro-Optics (CLEO), CFD2 (2005)

102. H. Dong, Y. Gong, V. Paulose, M. Hong, Opt. Commun. 282, 3671 (2009)

103. H. Dong, Y. Gong, M. Olivo, Microw. Opt. Technol. Lett. 52, 2319 (2010)

104. C. M. Morris, R. Valdés Aguilar, A. V. Stier, N. P. Armitage, Opt. Express 20, 12303 (2012)

105. S. R. Doshi, J. M. Charrier, Polym. Comp. 10, 28 (1989)

106. S.-Y. Fu, B. Lauke, Comp. Sci. Technol. 56, 1179 (1996)

107. D. Stifter, K. Wiesauer, M. Wurm, E. Schlotthauer, J. Kastner, M. Pircher, E. Götzinger, C. K. Hitzenberger, Meas. Sci. Technol. 19, 074011 (2008)

108. D. K. Hsu, K.-S. Lee, J.-W. Park, Y.-D. Woo, K.-H. Im, Internat. J. of Precision Eng. and Manufact. 13, $1183(2012)$

109. K.-H. Im, D. K. Hsu, C.-P. Chiou, D. J. Barnard, I.-Y. Yang, J.-W. Park, Review of Progress in Quantitative Nondestructive Evaluation, AIP Conf. Proc. 1430, 1192 (2012)

110. J.-W. Park, K.-H. Im, D. K. Hus, J.-A. Jung, I.-Y. Yang, J. Meas. Sci. Technol. 26, 2051 (2012)

111. S. Katletz, M. Pfleger, H. Pühringer, N. Vieweg, B. Scherger, B. Heinen, M. Koch, K. Wiesauer, Opt. Express 19, 23042 (2011)

112. Y. Hirota, R. Hattori, M. Tani, M. Hangyo, Opt. Express 14, 4486 (2006) 